\title{
RHABDITID SPECIES (NEMATODA, RHABDITIDA) RECORDED IN PENINSULAR SPAIN AND BALEARIC ISLANDS
}

\author{
J. Abolafia $(*)$ and R. Peña-Santiago $(*)$
}

\begin{abstract}
SUMMARY
Previous records of species belonging to the order Rhabditida in peninsular Spain and Balearic Islands is presented as compilation. Eighty species of forty genera and twelve families are listed. Information concerning each species contains scientific name, synonymy, distribution and remarks. Distributional data include localities and/or geographical areas where the species have been collected and the corresponding references. A short discussion on current knowledge on rhabditid nematodes in the Iberian geography is also made. Majority of species are defficiently known, being necessary additional taxonomic and/or faunistic studies of this taxon.
\end{abstract}

Keywords: Compilation, distribution, Iberian fauna, rhabditid nematodes, records.

\section{RESUMEN}

\section{Especies de rhabdítidos (Nematoda: Rhabditida) citadas en España} peninsular y las Islas Baleares

Se presenta una recopilación de citas previas de especies pertenecientes al orden Rhabditida en la España peninsular e Islas Baleares. Se listan ochenta especies de cuarenta géneros y doce familias. La información que concierne a cada especie contiene el nombre científico, sinonimia, distribución y observaciones. Los datos de distribución incluyen las localidades y/o áreas geográficas donde las especies han sido recolectadas, y las referencias correspondientes. También se realiza una breve discusión sobre el conocimiento de los nematodos rhabdítidos en el área ibérica, concluyéndose que la mayor parte de las especies se conocen deficientemente, siendo necesario un estudio taxonómico y/o faunístico adicional del taxon.

Palabras clave: Citas, distribución, fauna ibérica, nematodos rhabdítidos, recopilación.

\section{Introduction}

Members of the order Rhabditida are one of the most important taxa within the phylum Nematoda. They are very abundant and widely distributed in both soil and freshwater, where exploit different food resources: saprophagous and/or bacteriovorous (cephalobid species), predators (diplogasterid species), insect-parasites or entomopathogens (stei- nernematid species) and even, if we take into account the new concept of the group by De Ley and Blaxter (in press), plant-parasites (tylenchs). Rhabditid nematodes are also a highly diversified group, currently including 142 genera ( $c f$. De Ley et al., 1998).

Relatively scarce information on rhabditid nematodes from Spain is available and no monographic study of the group has been previously

* Departamento de Biología Animal, Biología Vegetal y Ecología, Universidad de Jaén. Campus "Las Lagunillas" s/n, Edificio B3. 23071-Jaén, Spain. Email: rpena@ujaen.es 
carried out. However, a small and rather disperse volume of data is found in numerous contributions dealing with general nematode fauna, or in a few articles containing descriptions of previously known and/or new species. This paper, which is compiling the general chorological information (species, localities, references) of Spanish rhabditid species as first source of need information, is conceived as a starting point for a detailed taxonomic study of the group in Andalucía Oriental (southeastern Spain).

Previous remarks should be pointed out. (i) Only records from peninsular Spain and Balearic Islands have been compiled. (ii) Just the information appeared in papers, book articles or books has been taken into account, and not so those records found in abstracts of congress, Ph. D. Thesis, etc. (iii) Those records containing specific identifications of the taxa have been compiled, but not so others in which just generic identification of specimens is given. (iv) No taxonomic study of specimens has been undertaken for this contribution, and, as consequence, data and remarks provided are based on the available literature. (v) In general, the modern - although already classic - Andrássy's $(1983,1984)$ classification is followed for higher categories, but some more recent nomenclatorial changes have been also introduced, mainly at generic and specific levels. (vi) References to taxa authorities are omitted in the corresponding list, i.e., only those papers refering to records and their remarks are presented.

\section{Historical outline}

Although the first records of rhabditid nematodes from Spain were made by Madrid-Moreno (1911), who cited Anguillulina stercorarius in two different habitats (a garden from Madrid city and plancton from Lozoya River), and Muñoz-Medina (1945) who found Rhabditis aspera in Sierra Nevada mountains (province of Granada), the pioner of nematological studies in Spain and the first author providing morphological and chorological information of rhabtidid species was Enrique Gadea Buissan. In his Ph. D. Thesis (1952), Gadea described and recorded eleven species, and in his numerous subsequent papers he would add new species and records to the Spanish nematode fauna. In the sixties, Jiménez-Millán and collaborators (see, for instance, Arias et al., 1963; Jiménez-Millán et al., 1965) reported additional records, even describing the new genus Chitinotylenchoides Arias \&
Jiménez-Millán, 1968 which is later on considered a junior synonym of Tylopharynx De Man, 1876. Jiménez-Guirado (1974, 1976) and Palomo (1979) made new contributions in the seventies. In the $80 \mathrm{~s}$ and 90s, Hernández et al. (1988), Hernández and Jordana (1990) and De Ley et al. (1994) recorded and described several rhabditid species, mainly cephalobs. Ocaña and Picazo (1991) carried out an interesting contribution to our knowledge of the species inhabiting freshwater in Sierra Nevada mountains. Finally, Navarro and Lluch (1999) have described a new species belonging to the genus Paracrobeles Heyns, 1968 from eastern Spain.

\section{Commented compendium of previous records of rhabditid nematode species from peninsular Spain and Balearic Islands}

The available information on the presence of rhabditid nematodes in Spain is compiled below. Species are alphabetically ordered according to their specific epithet. Data of each species contains four sections: scientific name, synonymy, distribution and remarks. Information concerning distribution includes the name of the localities where the species was found, the province of these localities (locality/province) and the respective reference. Appendix I shows the taxonomic position of the species, and Appendix II offers an alphabetical list of the species ordered by their generic names.

aspera, Rhabditis Bütschli, 1873

REMARKS: See under its senior synonym $R$. terricola Dujardin, 1845.

axei, Rhabditis (Cobbold, 1884) Dougherty, 1955

SYNONYMY: Pelodera axei Cobbold, 1884; Rhabditella axei (Cobbold, 1884) Chitwood, 1933.

Distribution: Quinto Real/Navarra (Monreal \& Campoy, 1982).

REMARKS: Monreal and Campoy (1982) provided measurements and illustrations of the material collected in Navarra. Andrássy (1984) considered this taxon as incertae sedis.

bernensis, Paroigolaimella (Steiner, 1914) Andrássy, 1958

Synonymy: Diplogaster bernensis Steiner, 1914; Diplogaster (Paradiplogaster) bernensis Steiner, 1914 (Hirschmann, 1952); Diplogaster (Paroigolaimella) bernensis Steiner, 1914 (Weintgärtner, 1955). 
DistRIBUTION: Sierra Nevada /Granada (Ocaña \& Picazo, 1991; Picazo et al., 1988).

REMARKS: No additional information about this species is available.

bisimilis, Heterocephalobus (Thorne, 1925) Andrássy, 1967

SYNONYMY: Acrobeles (Acrobeles) bisimilis Thorne, 1925; Eucephalobus bisimilis (Thorne, 1925) Thorne, 1937; Chiloplacus bisimilis (Thorne, 1925) Goodey, 1963; Acrobeloides karakalpakensis Karimova, 1957.

DistRiBUTION: Sierra Nevada/Granada (Ocaña and Picazo, 1991; Picazo et al., 1988); Beunza and Sansoain/Navarra (Hernández et al., 1988; Hernández \& Jordana, 1990 \& 1992).

REMARKS: No additional information about this species is available.

buetschlii, Acrobeloides (De Man, 1884) Steiner et Buhrer, 1933

Synonymy: Cephalobus buetschlii De Man, 1884; Cephalobus (Acrobeles) buetschlii De Man, 1884 (De Man, 1921); Acrobeles (Acrobeloides) buetschlii (De Man, 1884) Thorne, 1925; Cephalobus persegnis buetschlii De Man, 1884 (Micoletzky, 1922); Acrobeloides recurvatus Goodey, 1963.

Distribution: Peña Redonda/León (Gadea, 1953b); Tagomago Island/Baleares (Gadea, 1954); Canfranc/Huesca (Gadea, 1956); Pitiusas Islands/Baleares (Gadea, 1969); Sierra de Gata/Salamanca (Palomo, 1979); Catalonian Central Pyrenean (Gadea, 1982); Quinto Real /Navarra (Monreal \& Campoy, 1982); Peñas de Echauri/Navarra (Mateo \& Campoy, 1983); Bardenas/Navarra (Jordana et al., 1987); Beunza, Sansoain and Unzué/Navarra (Hernández et al., 1988; Hernández \& Jordana, 1990 \& 1992); Sierra of Grazalema/Cádiz (Santos Lobatón, 1991); Sierra Morena/Sevilla (Santos-Lobatón, 1992).

REMARKS: Monreal and Campoy (1982) and Mateo and Campoy (1983) provided measurements and/or illustrations of the material collected in Navarra.

carinata, Oligolaimella Zullini, 1981

Distribution: Sierra Nevada/Granada (Picazo et al., 1988; Ocaña \& Picazo, 1991).

REMARKS: No additional information about this species is available.

cervus, Cervidellus (Thorne, 1925) Thorne, 1937

SyNONymy: Acrobeles cervus Thorne, 1925
Distribution: Sierra de Gata/Salamanca (Palomo, 1979).

REMARKS: No additional information about this species is available.

ciliatus, Acrobeles von Linstow, 1877

Synonymy: Cephalobus ciliatus (von Linstow, 1877) De Man, 1880; Cephalobus (Acrobeles) ciliatus De Man, 1880 (Micoletzky, 1922); Acrobeles sinensis Kreis, 1930; Acrobeles maeneenus Yeates, 1967.

Distribution: San Juan de la Peña/Huesca (Gadea, 1956); Sierras de Béjar and Gata/Salamanca (Palomo, 1979); Catalonian Central Pyrenean (Gadea, 1982); Añorbe and Ezprogui/Navarra (Armendáriz et al., 1996).

REMARKS: No additional information about this species is available.

complexus, Acrobeles Thorne, 1925

SynONymy: Seleborca complexa (Thorne, 1925) Andrássy, 1985; Acrobeles crossotus Steiner, 1929.

DistRIBUTION: Sierra Nevada/Granada (Jiménez-Guirado, 1976); Beunza/Navarra (Hernández \& Jordana, 1992).

REMARKS: Hernández and Jordana (1992) recor$\operatorname{ded} A$. crossotus, a junior synonym of $A$. complexus.

coomansi, Drilocephalobus Ali, Suryawanshi et Chisty, 1973

Distribution: Añorbe and Ezprogui/Navarra (Armendáriz et al., 1996)

REMARKS: No additional information about this species is available.

cornis, Eucephalobus (Thorne, 1925) Andrássy, 1967 SynONymy: Acrobeloides cornis Thorne, 1925; Cephalobus cornis (Thorne, 1925) Thorne, 1937; Chiloplacus cornis (Thorne, 1925) Goodey, 1963.

DistRiBution: Sierra Nevada/Granada (Picazo et al., 1988; Ocaña \& Picazo, 1991).

REMARKS: No additional information about this species is available.

coronatus, Diploscapter (Cobb, 1893) Cobb, 1913 SYNONYMY: Rhabditis coronata Cobb, 1893; Rhabditis bicornis Zimmermann, 1898; Diploscapter bicornis (Zimmermann, 1898) Goodey, 1963; Rhabditis cephaloboides Stefanski, 1922; Acrobeles armatus Kreis, 1929.

Distribution: Sierra Nevada/Granada (Picazo et al., 1988; Ocaña \& Picazo, 1991; Ocaña, 1992).

REMARKS: No additional information about this species is available. 
costatus, Teratocephalus Andrássy, 1958

SyNONYMy: Teratocephalus decarinus Anderson, 1969.

Distribution: Beunza and Unzué/Navarra (Hernández et al., 1988; Hernández \& Jordana, 1988, 1990 \& 1992); Sansoain/Navarra (Hernández et al., 1988); Beorburu, Ezprogui and Leoz/Navarra (Armendáriz et al., 1996).

REMARKS: Hernández and Jordana (1988) provided good description and illustrations of Spanish specimens found in the North of the country. Armendáriz et al. (1996) recorded this species as $T$. decarinus, a junior synonym of $T$. costatus.

crassidens, Metateratocephalus (De Man, 1880) Eroshenko, 1973

SYNONYMY: Teratocephalus crassidens De Man, 1880; Euteratocephalus crassidens (De Man, 1880) Andrássy, 1958; Teratocephalus spiralis Micoletzky, 1913; Metateratocephalus typicus Eroshenko, 1973; Euteratocephalus minor Mukhina, 1981.

Distribution: Santa Fe de Montseny/Barcelona, Mendaca-Elorrio/Vizcaya, Picos de Europa/Asturias (Gadea, 1952a); Benaoján and Sierra de las Nieves/Málaga (Gadea, 1953a); Picos de Europa and Tama/Asturias (Gadea, 1953b); Sierra de Guadarrama/Madrid (Gadea, 1953c); Engors/Gerona (Gadea, 1956); Alayor and Tomago Island/Baleares (Gadea, 1962a); Medas Islands (Gadea, 1964a); Santa Eulalia del Río/Ibiza-Baleares (Gadea, 1964b); Menorca/Baleares, Pitiusas Islands/Baleares (Gadea, 1969); Quinto Real/Navarra (Monreal \& Campoy, 1982); Montes Alberes/Gerona (Gadea, 1983); Beunza/Navarra (Hernández \& Jordana, 1992); Beorburu/Navarra (Armendáriz et al., 1996).

REMARKS: $M$. crassidens is a widely distributed species around the world. Some doubt persits on the identity of the material described by Gadea (1952) due to its measurements are out of the usual ranges of the species $(\mathrm{L}=0.84$ vs $\mathrm{L}=0.3-0.5 \mathrm{~mm} ; \mathrm{V}=44$ vs $\mathrm{V}=53-60 ; \mathrm{c}=11.3$ vs $\mathrm{c}=7-10)$. However, other specimens studied by Gadea (1953a) fit well with the available information of the species. Monreal and Campoy (1982) provided measurements and illustrations of the material collected in Navarra.

crossotus, Acrobeles Steiner, 1929

REMARKS: See under A. complexus, its senior synonym.

curvicaudata, Cephaloboides (Schneider, 1866) Zullini, 1982

SyNONYMY: Leptodera curvicaudata Schneider, 1866; Rhabditis curvicaudata (Schneider, 1866) von Linstow, 1878;
Rhabditis (Choriorhabditis) curvicaudata (Schneider, 1866) von Linstow, 1878 (Osche, 1952); Rhabditis (Curviditis) curvicaudata (Schneider, 1866) von Linstow, 1878 (Dougherty, 1953); Rhabditis (Cephaloboides) curvicaudata (Schneider, 1866) von Linstow, 1878 (Dougherty, 1955); Rhabditis micoletzkyi Schneider, 1923; Rhabditis insulana Ditlevsen, 1928; Rhabditis armata Fuchs, 1931; Rhabditis (Choriorhabditis) armata Fuchs, 1931 (Osche, 1952); Rhabditis (Cephaloboides) armata Fuchs, 1931 (Dougherty, 1955); Curviditis curvicaudata (Schneider, 1866) Andrássy, 1983.

Distribution: Sierra Nevada/Granada (Picazo et al., 1988; Ocaña \& Picazo, 1991).

REMARKS: No additional information about this species is available.

dadayi, Teratocephalus Andrássy, 1968

SynONYmY: Teratocephalus subvexus Anderson, 1969; Teratocephalus sigillarius Eroshenko, 1973.

Distribution: Beunza/Navarra (Hernández \& Jordana, 1988 \& 1992); Unzué/Navarra (Hernández et al., 1988; Hernández \& Jordana, 1990 \& 1992).

REMARKs: Hernández et al. (1988) and Hernández and Jordana (1990) recorded T. dadayi and T. subvexus from Navarra, but these species are synonymous. Hernández and Jordana (1988) provided good description and illustrations of Spanish specimens found in the North of the country.

decarinus, Teratocephalus Anderson, 1969

REMARKS: See under T. costatus, its senior synonym.

devimucronata, Stegelletina (Sumenkova, 1964) Boström \& De Ley, 1996

SynONYMY: Cervidellus devimucronatus Sumenkova, 1964; Ypsylonellus devimucronatus (Sumenkova, 1964) Andrássy, 1984.

Distribution: Sierra Nevada/Granada (Picazo et al., 1988; Ocaña \& Pizaco, 1991); Puerto del Pinar/Granada (De Ley et al., 1994).

REMARKS: De Ley et al. (1994) described in detail one female collected from an oak forest, and identified it as Ypsylonellus cf. devimucronatus due to doubts about the exact diagnosis of the species.

elaboratus, Acrobeles Thorne, 1925

Distribution: El Arahal/Sevilla (Arias et al., 1963); El Arahal and Marchena/Sevilla, and Madrid/ Madrid (Jiménez-Millán et al., 1965).

REMARKS: Unfortunately no description nor illustration of the species were given by the authors who recorded it, and no posterior finding is known. 
The presence of $A$. elaboratus in the Iberian Peninsula should be confirmed.

elongata, Rhabditis Schneider, 1866

REMARKS: See under Rhabditella pseudoelonga$t a$, its senior synonym.

elongatus, Heterocephalobus (De Man, 1880) Andrássy, 1967

Synonymy: Cephalobus elongatus De Man, 1880; Cephalobus (Eucephalobus) elongatus De Man, 1880 (Schneider, 1939); Eucephalobus elongatus (De Man, 1880) Thorne, 1937; Cephalobus mylakolaimus Fuchs, 1930; Eucephalobus mylakolaimus (Fuchs, 1930) Thorne, 1937; Cephalobus (Heterocephalobus) kaczanowskii Brzeski, 1960; Heterocephalobus kaczanowskii (Brzeski, 1960) Brzeski, 1961; Cephalobus multicinctus Cobb, 1983; Eucephalobus multicinctus (Cobb, 1893) Thorne, 1937; Heterocephalobus multicinctus (Cobb, 1983) Andrássy, 1967; Cephalobus bipapillatus Stefanski, 1915; Eucephalobus bipapillatus (Stefanski, 1915) Thorne, 1937; Heterocephalobus bipapillatus (Stefanski, 1915) Andrássy, 1967.

Distribution: Barcelona, Manresa and Monserrat/Barcelona, Engors, La Molina, Olot and San Miguel/Gerona, Monte Larrobe/Vizcaya, Panticosa/Huesca, Picos de Europa/Asturias, Deva River/Cantabria, and Sierra de Aralar/Navarra (Gadea, 1952a); Navas de San Luis/Málaga (Gadea, 1953a); Mampodre and Riaño/León, and Picos de Europa/Asturias (Gadea, 1953b); Sierra de Guadarrama (Gadea, 1953c); Panticosa and Turbón/Huesca and Engors/Gerona (Gadea, 1956); San Luis/Menorca-Baleares (Gadea, 1962a); Medas Islands (Gadea, 1964a); Santa Eulalia del Río/Ibiza-Baleares and Tagomago Island/Baleares (Gadea, 1964b); Menorca/Baleares, Pitiusas Islands//Baleares (Gadea, 1969); Sierra de Béjar/Salamanca (Palomo, 1979); Catalonian Central Pyrenean (Gadea, 1982); Quinto Real/Navarra (Monreal \& Campoy, 1982); Peñas de Echauri/Navarra (Mateo \& Campoy, 1983); Montes Alberes/Gerona (Gadea, 1983); Beunza and Unzué/Navarra (Hernández et al., 1988; Hernández \& Jordana, 1990 \& 1992); Sansoain/Navarra (Jordana et al., 1987; Hernández et al., 1988; Hernández \& Jordana, 1990 \& 1992); Añorbe, Beorburu and Ezprogui/Navarra (Armendáriz et al., 1996).

REMARKS: This is a cosmopolitan species, found mainly in terrestrial habitats, and apparently widely distributed in the Iberian Peninsula and Balearic Islands. Nevertheless, measurements of various Spanish specimens studied by Gadea (1952a, 1953a) differ from the usual range of the species: longer $(\mathrm{L}=1.21$ vs $\mathrm{L}=0.6-0.9 \mathrm{~mm})$ and more obese body ( $\mathrm{a}=17,18.5$ vs $\mathrm{a}=25-33)$ and more anterior vulva ( $\mathrm{V}=48$ vs $\mathrm{V}=57-65)$; then, some doubt persits on the precise identity of this material. Anyway, very recent records of the species seem to confirm that it is a member of the Spanish fauna. Monreal and Campoy (1982) and Mateo and Campoy (1983) provided measurements and/or illustrations of the material collected in Navarra.

emarginatus, Acrolobus (De Man, 1880) Boström, 1985

SyNONYMY: Cephalobus emarginatus de Man, 1880; Acrobeloides emarginatus (De Man, 1880) Thorne, 1937; Acrobeles (Acrobeloides) emarginatus (De Man, 1880) Schneider, 1939; Panagrobelus emarginatus (De Man, 1880) Andrássy, 1984.

Distribution: Montseny/Barcelona, Sierra de Guadarrama/Madrid, Deva River/Cantabria, Picos de Europa and Orzales/Cantabria, Puigmal Summit/Gerona, Malniu, Maranges and Ribas de Fresser/Gerona, and Mendaca-Elorrio/Vizcaya (Gadea, 1952a), Benaoján/Málaga (Gadea, 1953a), Mampodre/León and Picos de Europa/Asturias (Gadea, 1953b), Sierra de Guadarrama/Madrid (Gadea, 1953c), Porriño/Pontevedra (Gadea, 1955b); Panticosa/Huesca and Maranges and Engors/Gerona (Gadea, 1956); Fornells/MenorcaBaleares (Gadea, 1962a) Estany Llebreta/Lérida (Gadea, 1962b); Tagomago Island/Baleares (Gadea, 1964b); Montejo de la Sierra/Madrid (Jiménez-Millán et al., 1965); Menorca/Baleares (Gadea, 1969); Montes Cántabros (Gadea, 1979); Catalonian Central Pyrenean (Gadea, 1982).

REMARKS: A. emarginatus is a problematic species (cf. Andrássy, 1984: 232). Gadea (1952a) recorded and described it, but either his measurements were wrong (excessively long body, $\mathrm{L}=2.4$ vs $\mathrm{L}=0.6$; and quite long tail, $\mathrm{c}=7.4$ vs $\mathrm{c}=14$ ), or the identification was erroneous; other records by Gadea (1953a) agree well in its measurements with the available information of the species. JiménezMillán et al. (1965) recorded Acrobeloides marginatus, certainly a typographical error, but no additional information was supplied by these authors.

facetus, Panagrolaimus (Massey, 1971) Andrássy, 1984

Synonymy: Panagromacra faceta Massey, 1971

Distribution: Sierra Nevada/Granada (Picazo et al., 1988; Ocaña \& Picazo, 1991).

REMARKS: No additional information about this species is available. 
fictor, Fictor (Bastian, 1865) Paramonov, 1952

Synonymy: Diplogaster fictor Bastian, 1865; Diplogaster (Eudiplogaster) fictor (Bastian, 1865) Meyl, 1961; Mononchoides fictor (Bastian, 1865) Meyl, 1961; Eudiplogaster fictor (Bastian, 1865) Meyl, 1961; Koerneria fictor (Bastian, 1865) Zullini, 1974; Diplogaster fluviatilis De Man, 1880; Diplogaster elpatiewski Daday, 1906; Fictor elpatiewski (Daday, 1906) Paramonov, 1952; Diplogaster roszowskyi Stefanski, 1915; Fictor roszowski (Stefanski, 1915) Paramonov, 1952; Butlerius brevispiculatus SchuurmansStekhoven \& Teunissen, 1938; Diplogaster brevispiculata (Schuurmans-Stekhoven \& Teunissen, 1938) Lordello \& Zamith, 1959.

Distribution: Noguera River/Lérida and Salitja/Gerona (Gadea, 1952); Bohí/Lérida (Gadea, 1956); Catalonian Central Pyrenean (Gadea, 1982).

REMARKS: No additional information about this species is available. Ebsary (1986) considered this taxon as species inquirienda.

\section{filiformis, Protorhabditis (Bütschli, 1873)}

Sudhaus, 1976

SYNONYMY: Rhabditis filiformis Bütschli, 1873; Rhabditis (Choriorhabditis) filiformis Bütschli, 1873 (Osche, 1952); Rhabditis agilis von Linstow, 1876; Protorhabditis lengerkeni Paetzold, 1958.

Distribution: Manresa and Rajadell/Barcelona, and Ribas de Fresser/Gerona (Gadea, 1952a); Maranges/Gerona (Gadea, 1956); Binillautí/Menorca-Baleares (Gadea, 1962a); Carrión River/Palencia (Gadea, 1963); Santa Eulalia del Río/Ibiza-Baleares (Gadea, 1964b); Pitiusas Islands/Baleares (Gadea, 1969); Sierras de Béjar and Gata / Salamanca (Palomo, 1979); Sierra Nevada/Granada (Ocaña, 1992).

REMARKS: Material described by Gadea (1952a) has a quite long body compared with the usual range of the species $(\mathrm{L}=1.2 v s \mathrm{~L}=0.45-0.60 \mathrm{~mm})$, and other authors did not supply additional information of the species. Therefore, revision of this material and new collection of specimens are needed to confirm the presence of $P$. filiformis in the country.

foetida, Tylopharynx (Bütschli, 1874) Goffart, 1930 Synonymy: Aphelenchus foetidus Bütschli, 1874; Aphelenchus (Paraphelenchus) foetidus Bütschli, 1874 (Micoletzky, 1922); Tylopharynx striata De Man, 1876; Neodiplogaster cryptolaimus Paramonov, 1951; Chitinotylenchoides mediterranensis Arias \& Jiménez-Millán, 1968.

DisTRIBUTION: Alcanar/Tarragona and Cocentaína/Alicante (Arias \& Jiménez-Millán, 1968 \& 1973; Bello et al., 1973).

REMARKs: Arias and Jiménez-Millán (1968) described Chitinotylenchoides mediterranensis, a junior synonym of this species, and later (1973) provided a good description of the species, inluding illustrations and measurements.

goffarti, Rhodolaimus (Sachs, 1949) Andrássy, 1971 SYNONYmY: Bunonema (Stammeria) goffarti Sachs, 1949; Bunonema (Rhodolaimus) goffarti Sachs, 1949 (Ruhm, 1962); Stammeria goffarti (Sachs, 1949) Andrássy, 1958.

Distribution: Quinto Real/Navarra (Monreal \& Campoy, 1982).

REMARKS: Monreal and Campoy (1982) provided measurements and illustrations of the material collected in Navarra.

graciloides, Metadiplogaster (Skwarra, 1921) Andrássy, 1984

Synonymy: Diplogaster graciloides Skwarra, 1921; Diplogastrellus graciloides (Skwarra, 1921) Paramonov, 1952; Diplogaster inaequidens Paesler, 1946; Diplogaster (Metadiplogaster) inaequidens Paesler, 1946 (Hirschmann, 1952); Diplogaster (Diplogastrellus) inaequidens Paesler, 1946 (Weintgärtner, 1955); Diplogastrellus inaequidens (Paesler, 1946) Paramonov, 1952; Metadiplogaster inaequidens (Paesler, 1946) Meyl, 1961.

Distribution: Manresa/Barcelona and Sardón de Duero/Valladolid (Gadea, 1952a).

REMARKS: This species was recorded by Gadea (1952a) as M. inaequidens, a junior synonym of the taxon.

hygrophilus, Panagrolaimus Bassen, 1940

DistRiBUTION: Sierra Nevada/Granada (Picazo et al., 1988; Ocaña \& Picazo, 1991; Ocaña, 1992).

REMARKS: No additional information about this species is available.

inaequidens, Metadiplogaster (Paesler, 1946) Meyl, 1961

REMARKS: See under Metadiplogaster graciloides, the senior synonym of the species.

inermis, Rhabditoides (Schneider, 1866) Dougherty, 1955

SyNONYMY: Leptodera inermis Schneider, 1866; Rhabditis inermis (Schneider, 1866) von Linstow, 1878; Rhabditis (Telorhabditis) inermis (Schneider, 1866) von Linstow, 1876 (Osche, 1952); Rhabditis (Rhabditoides) inermis (Schneider, 1866) von Linstow, 1876 (Sudhaus, 1876); Rhabditis faecalis Watanabe, 1920; Rhabditis hominis Kobayashi, 1920; Rhabditis schachtiella Skrjabin \& Shults, 1926; Rhabditoides schachtiella (Skrjabin \& Shults, 1926) Dougherty, 1955; Rhabditis inermoides Völk, 1950; Rhabditis (Telorhabditis) inermoides Völk, 1950 (Osche, 1952); Rhitis inermis (Schneider, 1866) Andrássy, 1983. 
DistRiBution: Mahou/Menorca-Baleares (Gadea, 1962a); Irati/Navarra (Hernández \& Jordana, 1992).

REMARKS: No additional information about this species is available.

labiatus, Cephalobus (Ivanova, 1968) Andrássy, 1984 SyNonymy: Acrobeloides labiatus Ivanova, 1968.

Distribution: Sierra Nevada/Granada (Picazo et al., 1988; Ocaña \& Picazo, 1991).

REMARKS: No additional information about this species is available.

latus, Heterocephalobus (Cobb, 1906) Andrássy, 1967

Synonymy: Cephalobus latus Cobb, 1906; Eucephalobus latus (Cobb, 1906) Thorne, 1937.

Distribution: Sierra Nevada/Granada (Picazo et al., 1988; Ocaña \& Picazo, 1991).

REMARKS: No additional information about this species is available.

\section{Iheritieri, Pristionchus (Maupas, Paramonov, 1952}

Synonymy: Diplogaster lheritieri Maupas, 1919; Diplogaster (Pristionchus) lheritieri Maupas, 1919 (Völk, 1950); Diplogaster(Mesodiplogaster) lheritieri Maupas, 1919 (Hirschmann, 1951); Mesodiplogaster lheritieri (Maupas, 1919) Goodey, 1963; Diplogaster lheritieri stenostoma Hirschmann, 1951; Diplogaster(Pristionchus) lheritieri stenostoma Hirschmann, 1951 (Weingärtner, 1955); Diplogaster(Pristionchus) lheritieri eurystoma Hirschmann, 1951 (Weingärtner, 1955); Diplogaster horticola Fuchs, 1929; Pristionchus horticola (Fuchs, 1929) Paramonov, 1952; Pristionchus longicaudatus Kreis, 1932; Pristinonchus ottoi Paramonov, 1952; Diplogaster obscuridens SchuurmansStekhoven, 1951; Mononchoides obscuridens (SchuurmansStekhoven, 1951) Goodey, 1963; Paramonoviola rhagii Blinova \& Vosilite, 1976.

Distribution: Irati/Navarra (Hernández \& Jordana, 1992).

REMARKS: No additional information about this species is available.

\section{longicaudata, Rhabditis Bastian, 1865}

SYNONYMY: Rhabditis (Choriorhabditis) longicaudata Bastian, 1865 (Osche, 1952); Rhabditis longicaudata Bütschli, 1873.

Distribution: Panjón/ Pontevedra (Gadea, 1955b); Sierra de Gata (Palomo, 1979); Ezprogui and Leoz/ Navarra (Armendáriz et al., 1996).

REMARKs: Armendáriz et al. (1996) recorded $R$. longicaudatula Bastian, 1865, certainly a typographical error. No additional information about this species is available. loofi, Pseudacrobeles (Bunobus) (Andrássy, 1968) De Ley, Siddiqi \& Boström, 1993

SyNONYMY: Heterocephalobus loofi Andrássy, 1968.

Distribution: Ezprogui/Navarra (Armendáriz et al., 1996).

REMARKS: No additional information about this species is available.

maupasi, Rhabditis Seurat in Maupas, 1919

SYNONYMY: Rhabditis (Choriorhabditis) maupasi Seurat in Maupas, 1919 (Osche, 1952); Rhabditis johnsoni Micoletzky, 1922; Rhabditis (Choriorhabditis) johnsoni Micoletzky, 1922 (Osche, 1952); Rhabditis leptodera Hertwig, 1922; Rhabditis (Choriorhabditis) leptodera Hertwig, 1922 (Osche, 1952).

Distribution: Mendaca-Elorrio/Vizcaya and Olot/Gerona (Gadea, 1952a).

REMARKS: Gadea (1952a) recorded and described this species from two localities in the North of the Iberian Peninsula but some of the measurements given by the Catalonian author are out of the usual range of the species $(\mathrm{L}=1.44 v s \mathrm{~L}=1.9$ $2.1 \mathrm{~mm} ; \mathrm{c}=14.4 v s \mathrm{c}=19-20$ ). Taking into account such differences and the fact that no posterior record of the species has been registered, some doubt persists of its belonging to the Iberian fauna.

minimus, Chiloplacus (Thorne, 1925) Andrássy, 1959

SynONYMY: Acrobeles (Acrobeloides) minimus Thorne, 1925; Cervidellus minimus (Thorne, 1925) Goodey, 1963; Acrobelophis minimus (Thorne, 1925) Andrássy, 1984; Acrobeles soosi Andrássy, 1953; Chiloplacus soosi (Andrássy, 1953) Andrássy, 1959; Cervidellus soosi (Andrássy, 1953) Goodey, 1963.

DistRiBUtion: Unzué/Navarra (Hernández et al., 1988; Hernández \& Jordana, 1990; Hernández \& Jordana, 1992); Añorbe and Ezprogui/Navarra (Armendáriz et al., 1996).

REMARKS: No additional information about this species is available.

mucronatus, Eucephalobus (Kozlowska \& Roguska-Wasilewska, 1963) Andrássy, 1967

SyNONYmy: Cephalobus mucronatus Kozlowska \& RoguskaWasilewska, 1963.

Distribution: Sansoain and Unzué/ Navarra (Jordana et al., 1987; Hernández et al., 1988; Hernández \& Jordana, 1990 \& 1992); Añorbe/Navarra (Armendáriz et al., 1996).

REMARKS: No additional information about this species is available. 
multipapillatum, Bunonema Stefanski, 1914

DistriBution: Añorbe, Beorburu, Eslava, Ezprogui, Javerri, Leoz, Marcaláin, Sabaiza and Zariquieta/ Navarra (Armendáriz \& Hernández, 1992; Armendáriz et al., 1994).

REMARKS: Armendáriz and Hernández (1992) provided measurements and illustrations of the specimens collected in northern Spain.

nanus, Acrobeloides (De Man, 1880) Anderson, 1968 SynONymy: Cephalobus nanus De Man, 1880; Cephalobus persegnis nanus De Man, 1880 (Micoletzky, 1922); Acrobeles (Acrobeloides) minor Thorne, 1925; Acrobeloides minor (Thorne, 1925) Thorne, 1937.

Distribution: Puigmal Summit/Gerona, Jaca/Huesca, Montseny/Barcelona, Deva River/Cantabria, Noguera River/Lérida and Sierra of Aralar/Navarra (Gadea, 1952a); Picos de Europa/Asturias (Gadea, 1953b); Canfranc, Selva de Oza and Panticosa/Huesca and Bohí/Lérida (Gadea, 1956); Alayor and La Falconera/Menorca-Baleares (Gadea, 1962a); Tagomago Island/Baleares (Gadea, 1964b); Catalonian Central Pyrenean (Gadea, 1982); Quinto Real/Navarra (Monreal \& Campoy, 1982); Peñas de Echauri/Navarra (Mateo \& Campoy, 1983); Bardenas/Navarra (Jordana et al., 1987); Beunza, Sansoain and Unzué/Navarra (Hernández et al., 1988; Hernández \& Jordana, 1990 \& 1992); Sierra Nevada/Granada (Ocaña, 1992); Sierra Morena/Sevilla (Santos-Lobatón, 1992); Añorbe, Beorburu, Ezprogui and Leoz/Navarra (Armendáriz et al., 1996).

REMARKS: This is a worldwide distributed species. Although some measurements provided by Gadea (1952) are out the usual range of the species $(\mathrm{V}=47$ vs $\mathrm{V}=62-68)$, more recent records confirm its presence in the North of the Iberian Peninsula and surely in the Balearic Islands. Monreal and Campoy (1982) and Mateo and Campoy (1983) provided measurements and/or illustrations of the material collected in Navarra.

neftasiensis, Cervidellus Boström, 1986

DisTRIBUTION: Sayerri Valley/Huesca (De Ley et al., 1994).

REMARKS: Good description and illustrations of the population collected in the province of Huesca were provided by De Ley et al. (1994).

nudicapitatus, Diplogasteritus (Steiner, 1914) Paramonov, 1952

SYNONYMY: Diplogaster nudicapitata Steiner, 1914; Acrostichus nudicapitatus (Steiner, 1914) Massey, 1962;
Diplogaster rhodani Stefanski, 1914; Diplogasteritus rhodani (Stefanski, 1914) Paramonov, 1952; Achrostichus rhodani (Stefanski, 1914) Massey, 1966.

DistRIBUTION: Sierra Nevada/Granada (Picazo et al., 1988; Ocaña \& Picazo, 1991; Ocaña, 1992).

REMARKS: No additional information about this species is available.

oxycerca, Poikilolaimus (De Man, 1895) Sudhaus, 1980

SYNONYMY: Rhabditis oxycerca De Man, 1895; Rhabditis (Choriorhabditis) oxycerca De Man, 1895 (Osche, 1952); Rhabditis (Cephaloboides) oxycerca De Man, 1895 (Dougherty, 1955); Cephaloboides oxycercus (De Man, 1895) Zullini, 1982; Anguillula brevispina Claus, 1862 (nomem oblitum !); Rhabditis brevispina (Claus, 1862) Bütschli, 1873; Rhabditis (Choriorhabditis) brevispina (Claus, 1862) Osche, 1952; Rhabditis nudicapitata Stefanski, 1922; Rhabditis demani Hnatewytsch, 1929; Rhabditis (Choriorhabditis) demani Hnatewytsch, 1929 (Meyl, 1954); Rhabditis variabilis Fuchs, 1931; Cuticularia mathesoni Van der Linde, 1938; Rhabditis mathesoni (Van der Linde, 1938) Goodey, 1951; Rhabditis (Choriorhabditis) mathesoni (Van der Linde, 1938) Goodey, 1951 (Dougherty, 1953); Rhabdtis stalbergi Allgén, 1950; Rhabditis oerleyi Völk, 1950; Rhabditis succaris Clapham, 1930; Rhabditis (Choriorhabditis) succaris Clapham, 1930 (Osche, 1952), Rhabditis parapapillosa Schuurmans Stekhoven, 1951; Peplorhabditis vestibularis Ivanova, 1960; Protorhabditis cuneocaudata Slepetiene, 1961; Praeputirhabditis jodhapurensis Khera, 1969; Rhabditis (Cephaloboides) jodhapurensis (Khera, 1969) Sudhaus, 1976; Cuticularia oxycerca (De Man, 1895) Andrássy, 1983.

DistRIBUTION: Sierra Nevada/Granada (Picazo et al., 1988; Ocaña \& Picazo, 1991; Ocaña, 1992).

REMARKS: No additional information about this species is available.

oxyuroides, Eucephalobus (De Man, 1876) Steiner, 1936

Synonymy: Cephalobus oxyuroides De Man, 1876; Cephalobus oxyuroides acuticaudatus Micoletzky, 1922; Cephalobus oxyuroides brasiliensis Rahm, 1928; Cephalobus similis Cobb, 1893.

Distribution: Binillautí and Mahón/MenorcaBaleares (Gadea, 1962a); Medas Islands (Gadea, 1964a); Sierra Nevada/Granada (Jiménez Guirado, 1976b); Sierras de Gata and Béjar/ Salamanca (Palomo, 1979); Quinto Real/Navarra (Monreal \& Campoy, 1982); Peñas de Echauri/Navarra (Mateo \& Campoy, 1983); Añorbe, Eslava, Ezprogui, Javerri, Marcaláin, Sabaiza and Zariquieta/Navarra (Armendáriz \& Hernández, 1994); Beorburu and Leoz/Navarra (Armendáriz and Hernández, 1994; Armendáriz et al., 1996); Beunza, Sansoain and Unzué/ Navarra (Hernández et al., 1988; Hernández \& Jordana, 1990 \& 1992). 
REMARKs: Monreal and Campoy (1982) and Mateo and Campoy (1983) provided measurements and/or illustrations of the material collected in Navarra.

palustris, Teratocephalus (De Man, 1880) Andrássy, 1958

SynONYMY: Teratocephalus palustris De Man, 1880; Teratocephalus palustris crassicauda Daday, 1897; Euteratocephalus palustris crassicauda (De Man, 1880) Meyl, 1961; Teratocephalus spiraloides Micoletzky, 1913; Euteratocephalus spiraloides (Micoletzky, 1913) Heyns, 1977; Teratocephalus cornutus Cobb, 1914; Teratocephalus palustris cornutus Cobb, 1914 (Micoletzky, 1922).

Distribution: Sierra Segundera/Zamora (Gadea, 1954b); Antela/Orense (Gadea, 1955b).

REMARKS: Short description and illustrations of Spanish specimens provided by Gadea (1954b) fit well with those of other populations, although their body is a bit shorter $(\mathrm{L}=0.6,0.9$ vs $\mathrm{L}=0.8-1.0 \mathrm{~mm})$ and the vulva more posterior $\left(\mathrm{V}=55,57 v_{\mathrm{S}} \mathrm{V}=50-52\right)$.

papillosa, Phasmarhabditis (Schneider, 1866) Andrássy, 1976

Synonymy: Pelodera papillosa Schneider, 1866; Rhabditis papillosa (Schneider, 1866) Örley, 1880; Rhabditis (Choriorhabditis) papillosa (Schneider, 1866) Örley, 1880 (Osche, 1952); Rhabditis (Pellioditis) papillosa (Schneider, 1866) Örley, 1880 (Dougherty, 1955); Rhabditis ikedai Tadano, 1950.

Distribution: Cueva Calera/Logroño (Gadea, 1955a).

REMARKS: Gadea (1955a) described and illustrated this species, but no posterior record is known in our country.

paracornutus, Eucephalobus De Coninck, 1943

Distribution: Peñas de Echauri/Navarra (Mateo \& Campoy, 1983).

REMARKs: Mateo and Campoy (1983) described and illustrated the population collected in northern Spain.

parvus, Cephalobus Thorne, 1937

DistRIBUTION: Sansoain/Navarra (Hernández et al., 1988; Hernández \& Jordana, 1990 \& 1992).

REMARKS: No additional information about this species is available.

paucipapillata, Bursilla (Paetzold, 1955) Andrássy, 1983

SYNONYMY: Rhabditis (Mesorhabditis) paucipapillata Paetzold, 1955; Mesorhabditis paucipapillata (Paetzold, 1955) Paetzold, 1958.
DistRIBUTION: Beunza and Sansoain/Navarra (Hernández et al., 1988; Hernández \& Jordana, 1990 \& 1992).

REMARKs: No additional information about this species is available.

\section{pellio, Pellioditis (Schneider, 1866) Timm, 1960}

SynONymy: Pelodera pellio Schneider, 1866; Rhabditis pellio (Schneider, 1866) Bütschli, 1873; Rhabditis (Choriorhabditis) pellio (Schneider, 1866) Bütschli, 1873 (Osche, 1952); Rhabditis (Pellioditis) pellio (Schneider, 1866) Bütschli, 1873 (Dougherty, 1953); Leptodera pellio (Schneider, 1866) Ward, 1903.

Distribution: Ciudadela/Menorca-Baleares (Gadea, 1962a).

REMARKS: No additional information about this species is available.

pellioides, Pellioditis (Bütschli, 1873) Andrássy, 1983

SYNONYMY: Rhabditis pellioides Bütschli, 1873; Rhabditis (Choriorhabditis) pellioides Bütschli, 1873 (Osche, 1952); Rhabditis oncomelaniae Yokoo \& Okabe, 1968; Rhabditis (Choriorhabditis) oncomelaniae Yokoo \& Okabe, 1968 (Sudhaus, 1976).

Distribution: Quinto Real/Navarra (Hernández \& Jordana, 1992)

REMARKS: No additional information about this species is available.

penardi, Bunonema Stefanski, 1914

Distribution: Irati/Navarra (Hernández \& Jordana, 1992).

REMARKS: No additional information about this species is available.

\section{persegnis, Cephalobus Bastian, 1865}

SYNONYMY: Cephalobus brevicaudatus Zimmermann, 1898; Cephalobus persegnis brevicaudatus Zimmermann, 1898 (Micoletzky, 1922).

Distribution: Navas de San Luis/Málaga (Gadea, 1953a); Mampodre/León (Gadea, 1953b); Turbón/Huesca (Gadea, 1956); Alayor and Mahón/Baleares (Gadea, 1962a); Menorca/Baleares (Gadea, 1969); Sierra Nevada/Granada (Jiménez-Guirado, 1976b); Catalonian Central Pyrenean (Gadea, 1982); Montes Alberes/Lérida (Gadea, 1983); Bardenas/ Navarra (Jordana et al., 1987); Sansoain and Unzué/Navarra (Hernández et al., 1988; Hernández \& Jordana, 1990 \& 1992); Sierra Nevada/Granada (Ocaña, 1992); Añorbe, Beorburu, Ezprogui and Leoz/Navarra (Armendáriz et al., 1996). 
REMARKS: No additional information about this species is available.

producta, Rhabditis (Schneider, 1866) von Linstow, 1878

SYNONYMY: Leptodera producta Schneider, 1866; Rhabditis (Choriorhabditis) producta (Schneider, 1866) von Linstow, 1878 (Osche, 1952).

Distribution: Casteldefells/Barcelona and Olot/Gerona (Gadea, 1952a); Sierra de Albarracín/Teruel (Gadea, 1952b); Mampodre/León and Picos de Europa/Asturias (Gadea, 1953b); Sierra de Guadarrama/Madrid (Gadea, 1953c, 1979); Panticosa/Huesca (Gadea, 1956); Alayor, Binillautí and Mahón/Menorca-Baleares (Gadea, 1962a); Albarán/Murcia (Jiménez-Millán, 1962b; Navacerrada, 1975); Carrión River/Palencia (Gadea, 1963); Dunas de Mitjorn, Isla Grande de Santa Eulalia and Isla Malví/Baleares (Gadea, 1964b); Menorca/Baleares, Pitiusas Islands/Baleares (Gadea, 1969); Sierra de Albarracín/Teruel (Gadea, 1979); Catalonian Central Pyrenean (Gadea, 1982); Quinto Real/Navarra (Monreal \& Campoy, 1982); Montes Alberes/Gerona (Gadea, 1983).

REMARKs: Monreal and Campoy (1982) provided measurements and illustrations of the material collected in Navarra.

propinquus, Chiloplacus (De Man, 1921) Thorne, 1937

Synonymy: Cephalobus (Acrobeles) propinquus De Man, 1921; Acrobeles (Acrobeloides) propinquum (De Man, 1921) Thorne, 1925; Acrobeles (Acrobeloides) contractus Thorne, 1925; Cephalobus contractus (Thorne, 1925) Steiner, 1934.

Distribution: El Arahal/Sevilla (Arias et al., 1963; Jiménez-Millán et al., 1965); Peñas de Echauri/Navarra (Mateo \& Campoy, 1983).

REMARKs: Mateo and Campoy (1983) illustrated and provided measurements of a population collected in northern Spain.

psammophilus, Paracrobeles Navarro \& Lluch, 1999

Distribution: El Saler/Valencia (Navarro \& Lluch, 1999).

REMARKS: This is the second species of the genus and its finding in our country has a great biogeographic interest due to the another species, Paracrobeles laterellus Heyns, 1968, is only known from South Africa and Namibia.

pseudoelongata, Rhabditella (Micoletzky, 1913) Andrássy, 1983

SYNONYMY: Rhabditis pseudoelongata Micoletzky, 1913;
Rhabditis (Rhabditella) pseudoelongata Micoletzky, 1913 (Sudhaus, 1976); Leptodera elongata Schneider, 1866, nec Baird, 1858; Rhabditis elongata (Schneider, 1866) Bütschli, 1876; Rhabditis (Choriorhabditis) elongata (Schneider, 1866) Bütschli, 1876 (Osche, 1952); Rhabditis tenuicaudata Menzel \& Stefanski in Stefanski, 1917; Rhabditella tenuicaudata (Menzel \& Stefanski in Stefanski, 1917) Chitwood, 1933; Rhabditis usui Watanabe, 1927; Rhabditis gracilis Shingareva, Demidova \& Kudriavthev, 1928; Rhabditella gracilis (Shingareva, Demidova \& Kudriavthev, 1928) Chitwood, 1933; Rhabditis macrocerca Kreis \& Faust, 1933; Rhabditella macrocerca (Kreis \& Faust, 1933) Steiner, 1943; Rhabditis tricincta Paesler, 1946; Rhabditella multipara Li, 1951.

Distribution: Desfiladero de los Veyos and Picos de Europa/Asturias (Gadea, 1953b); Sierra de Gata/Salamanca (Palomo, 1979).

REMARKS: Spanish records of the species refer to Rhabditis elongata, a junior synonym of the species. No additional information about this species is available.

pseudolatus, Pseudacrobeles (Bunobus) (Hernández, 1990) De Ley, Siddiqi \& Boström, 1993

SynONYMY: Heterocephalobus pseudolatus Hernández, 1990.

Distribution: Beunza/Navarra (Hernández, 1990); Añorbe/Navarra (Armendáriz et al., 1996).

REMARKS: At present, this species is only known from Spain. Hernández (1990) provided a detailed description of it, including illustrations and measurements.

punctata, Pelodera (Cobb, 1914) Dougherty, 1955 SYNONYMY: Rhabditis punctata Cobb, 1914; Rhabditis (Pelodera) punctata Cobb, 1914 (Sudhaus, 1976); Rhabditis chitwoodi Bassen, 1940; Pelodera chitwoodi (Bassen, 1940) Dougherty, 1955; Teratorhabditis vivipara Gagarin, 1977.

DistRiBution: Sierra Nevada/Granada (Picazo et al., 1988; Ocaña \& Picazo, 1991).

REMARKS: No additional information about this species is available.

\section{pyrenaicus, Teratocephalus Gadea, 1956}

DistRIBUTION: Engors/Gerona (Gadea, 1956).

REMARKS: Gadea (1956: 144) proposed T. pyrenaicus as new species, but no description, measurements nor illustration were supplied by the Catalonian author. So, it must be considered as nomen nudum.

reticulatum, Bunonema Richters, 1905

SyNonYMY: Bunonema bogdanovi Zograf, 1913.

DistRIBUTION: Quinto Real/Navarra (Monreal \& Campoy, 1982). 
REMARKS: Monreal and Campoy (1982) provided measurements and illustrations of the material collected in Navarra.

richtersi, Bunonema Jägerskiöld, 1905

SYNONYMY: Bunonema richtersi aberrans Steiner, 1914.

DisTRIBUTION: Quinto Real/Navarra (Monreal \& Campoy, 1982); Beunza/Navarra (Hernández et al., 1988; Hernández \& Jordana, 1990 \& 1992; Armendáriz \& Hernández, 1992); Sansoain /Navarra (Hernández et al., 1988; Armendáriz \& Hernández, 1992); Beorburu/Navarra (Armendáriz et al., 1996).

Remarks: Monreal and Campoy (1982) and Armendáriz and Hernández (1992) provided measurements and illustrations of the specimens collected in northern Spain.

rigidus, Panagrolaimus (Schneider, 1866) Thorne, 1937

SynONYMY: Leptodera rigida Schneider, 1866; Anguillula rigida (Schneider, 1866) Bütschli, 1876; Cephalobus rigidus (Schneider, 1866) Örley, 1880; Rhabditis rigida (Schneider, 1869 von Linstow, 1883; Cephalobus oxyuris Bütschli, 1873; Panagrolaimus oxyuris (Bütschli, 1873) Goodey, 1963 Cephalobus infestans Cobb, 1893; Rhabditis aquatica Micoletzky, 1913; Panagrolaimus aquaticus (Micoletzky, 1913) Goodey, 1963.

Distribution: Barcelona and Montseny/Barcelona, Engors and Maranges/Gerona, and Peñalara/Madrid (Gadea, 1952a); Sierra de Albarracín/Teruel (Gadea, 1952b); Sierra de Guadarrama (Gadea, 1953c); Panticosa/Huesca and Maranges/Gerona (Gadea, 1956); Ciudadela /Menorca-Baleares (Gadea, 1962a); Malví Island/Baleares (Gadea, 1964b); Columbretes Islands (Gadea, 1974); Isla Mayor/Murcia (Gadea, 1976); Sierra Nevada/Granada (Jiménez-Guirado, 1976b); Catalonian Central Pyrenean (Gadea, 1982); Quinto Real/Navarra (Monreal \& Campoy, 1982); Montes Alberes/Gerona (Gadea, 1983); Sierra Nevada/Granada (Picazo et al., 1988; Picazo \& Ocaña, 1991); Quinto Real/Navarra (Hernández \& Jordana, 1992); Añorbe, Beorburu, Ezprogui and Leoz / Navarra (Armendáriz et al., 1996).

REMARKS: This is a cosmopolitan species, certainly widely distributed in our soils and freshwaters. Description and measurements of Spanish material provided by Gadea (1952a) agree well with other European populations although some small but significant differences can be noted: longer neck $(b=3.3$ vs $b=4.6-5.9)$ and more anterior vulva $(\mathrm{V}=51$ vs $\mathrm{V}=57-61)$. Then, it is convenient the study of additional Spanish material to confirm such differences which can have taxonomic interest. Some of the records by Gadea (e.g. 1956, 1982 in part) correspond to Cephalobus oxyuris, a junior synonym of $P$. rigidus. Monreal and Campoy (1982) provided measurements and illustrations of the material collected in Navarra.

rivalis, Diplogaster (Leydig, 1854) Bütschli, 1873

SynONyMY: Oncholaimus rivalis Leydig, 1854; Diplogaster micans Schultze in Carus, 1857; Diplogaster tridentata Metchnikov, 1863; Diplogaster vivipara von Linstow, 1879; Diplogaster macrodon Öerley, 1880; Diplogaster lacustris Daday, 1894; Diplogaster laevis de Cillis, 1917; Diplogaster bodamica Micoletzky, 1922.

Distribution: El Grao/Castellón (Gadea, 1952c); Maranges/Gerona (Gadea, 1956); Carrión River/Palencia (Gadea, 1963).

REMARKS: Gadea (1952c) described and illustrated in detail the specimens found in Castellón, and his measurements fit well with the available data of the species.

ruffoi, Mononchoides Zullini, 1983

DistRIBUTION: Sierra Nevada/Granada (Picazo et al., 1988).

REMARKS: No additional information about this species is available.

schneideri, Rhabditophanes (Bütschli, 1873) Goodey, 1953

SYNONYMY: Rhabditis schneideri Bütschli, 1873.

Distribution: Cubillas River/Granada (JiménezGuirado, 1974); Sierra de Gata (Palomo, 1979).

REMARKS: An interesting taxonomic contribution on this species was carried out by JiménezGuirado (1974), including description, measurements and illustrations of the material collected in the South of the Iberian Peninsula.

serratus, Cervidellus (Thorne, 1925) Thorne, 1937

REMARKs: See under Cervidellus vexilliger, its senior synonym.

similis, Stegelletina (Thorne, 1925) Boström \& De Ley, 1996

SynONYMY: Acrobeles similis Thorne, 1925; Cervidellus similis (Thorne, 1925) Thorne, 1937; Ypsylonellus similis (Thorne, 1925) Andrássy, 1984; Stellegetina lineata (Thorne, 1925) Andrássy, 1984; Stellegetina mucronata (Loof, 1971) Andrássy, 1984.

Distribution: Puerto del Pinar/Granada, Igüer and Sayerri Valleys/Huesca (De Ley et al., 1994). 
REMARKS: De Ley et al. (1994) published a very detailed description of this species, including SEM illustrations.

\section{singulus, Acrobeles Heyns, 1969}

Distribution: Unzué/Navarra (Hernández et al., 1988; Hernández \& Jordana, 1990 \& 1992); Bardenas/Navarra (Jordana et al., 1987).

REMARKS: Andrássy (1984) considered this species a junior synonym of A. ciliatus, but after Shahina and De Ley (1997) it is valid. No additional information about this species is available.

skrjabini, Acromoldavicus (Nesterov \& Lisetskaya, 1965) Nesterov, 1970

SynonymY: Acrobeloides skrjabini Nesterov \& Lisetskaya, 1965.

Distribution: Sierra de la Sagra/Granada (Karegar et al., 1997).

REMARKS: Karegar et al. (1997) studied three specimens of this species from Spain and provided a very detailed description of this material including SEM photographs.

soosi, Acrobelophis (Andrássy, 1953) Andrássy, 1984

REMARKs: See under Chiloplacus minimus, its senior synonym.

spiculigera, Mesorhabditis (Steiner, 1936) Dougherty, 1953

SYNONYMY: Rhabdtis spiculigera Steiner, 1936; Rhabditis (Mesorhabditis) spiculigera Steiner, 1936 (Osche, 1952); Pseudorhabditis acuminata Kreis, 1929; Asymmetricus acuminatus (Kreis, 1929) Kreis, 1930; Rhabditis acuminata (Kreis, 1929) Nigon, 1949; Tricephalobus acuminatus (Kreis, 1929) Goodey, 1951; Mesorhabditis acuminata (Kreis, 1929) Dougherty, 1955; Trilabiatus acuminatus (Kreis, 1929) Goodey, 1963.

Distribution: Sierra de Gata/Salamanca (Palomo, 1979); Quinto Real/Navarra (Monreal \& Campoy, 1982); Beunza/Navarra (Hernández et al., 1988); Sansoain/Navarra (Hernández et al., 1988; Jordana et al., 1987); Añorbe, Beorburu, Ezprogui and Leoz/Navarra (Armendáriz et al., 1996).

REMARKs: Monreal and Campoy (1982) provided measurements and illustrations of the material collected in Navarra. Jordana et al. (1987) recorded Mesorhabditis cf. spiculigera from Sansoain, province of Navarra.

stigmatus, Rhabditidoides (Steiner, 1930) Andrássy, 1984

Synonymy: Diplogasteroides stigmatus Steiner, 1930; Diplogasteroides (Anchidiplogasteroides) stigmatus Steiner, 1930 (Körner, 1954); Diplogasteroides (Rhabdontolaimus) stigmatus Steiner, 1930 (Rühm, 1956); Rhabditolaimus stigmatus (Steiner, 1930) Andrássy, 1958; Rhabdontolaimus stigmatus (Steiner, 1930) Meyl, 1961; Anchidiplogasteroides stigmatus (Steiner, 1930) Paramonov, 1964.

Distribution: Sierra Nevada/Granada (Picazo et al., 1988; Ocaña \& Picazo, 1991).

REMARKS: No additional information about this species is available.

striatus, Eucephalobus (Bastian, 1865) Thorne, 1937 Synonymy: Cephalobus striatus Bastian, 1865; Cephalobus (Eucephalobus) striatus Bastian, 1865 (Schneider, 1939); Cephalobus striatus atubifer Micoletzky, 1922; Cephalobus striatus tubifer Micoletzky, 1922; Cephalobus striatus microtubifer Micoletzky, 1922; Cephalobus bursifer De Man, 1876.

Distribution: Sierra Nevada/Granada (Picazo et al., 1988; Ocaña \& Picazo, 1991); Añorbe, Ezprogui and Leoz/Navarra (Armendáriz et al., 1996); Sierra Bermeja/Málaga, Sierra de las Nieves/Málaga and Sierra de Grazalema/Cádiz (Peña-Santiago et al., 1996).

REMARKS: No additional information about this species is available.

strongyloides, Pelodera (Schneider, 1860) Schneider, 1866

SYNONYMY: Pelodytes strongyloides Schneider, 1866; Leptodera strongyloides (Schneider, 1866) Schneider, 1866; Rhabditis strongyloides (Schneider, 1860) von Linstow, 1878; Rhabditis (Pelodera) strongyloides (Schneider, 1866) von Linstow, 1878 (Sudhaus, 1976); Rhabditis teroides Micoletzky, 1915; Rhabdtis taurica Mirethkij \& Skrjabin, 1865; Pelodera comandorica Belogurov, Mukhina \& Churikova, 1977.

Distribution: Sierra de Gata/Salamanca (Palomo, 1979).

REMARKS: No additional information about this species is available.

subvesus, Teratocephalus Anderson, 1969

REMARKS: See under T. dadayi, its senior synonym.

symmetricus, Chiloplacus (Thorne, 1925) Thorne, 1937

Synonymy: Acrobeles (Acrobeloides) symmetricus Thorne, 1925; Cephalobus symmetricus (Thorne, 1925) Steiner, 1935.

Distribution: Unzué/Navarra (Hernández et al., 1988; Hernández \& Jordana, 1990 \& 1992).

REMARKS: No additional information about this species is available.

teres, Pelodera Schneider, 1866

SyNONYMY: Leptodera teres (Schneider, 1866) Schneider, 1866; Rhabditis teres (Schneider, 1866) Bütschli, 1873; 
Rhabditis (Pelodera) teres (Schneider, 1866) Bütschli, 1873 (Sudhaus, 1976); Aguillula mucronata Grube, 1849; Rhabditis mucronata (Grube, 1849) Bastian, 1865; Pelodera mucronata (Grube, 1849) Schneider, 1866; Rhabditis donbass Skrjabin, Schults \& Serbinov, 1926; Pelodera donbass (Skrjabin, Schults \& Serbinov, 1926) Dougherty, 1955.

Distribution: Mendaca-Elorrio/Vizcaya, Peñalara/Cantabria, Felanix/Mallorca-Baleares, Jaca, Panticosa and Selva de Oza/Huesca, and Castelldefels/Barcelona (Gadea, 1952a); Isla Mayor/Murcia (Gadea, 1976).

REMARKS: See comments under $R$. terricola.

teres, Pseudacrobeles (Pseudacrobeles) (Thorne, 1937) De Ley, Siddiqi \& Boström, 1993

Synonymy: Eucephalobus teres Thorne, 1937; Cephalobus (Eucephalobus) teres (Thorne, 1937) Rühm, 1956; Heterocephalobus teres (Thorne, 1937) Andrássy, 1967.

Distribution: Quinto Real/Navarra (Monreal \& Campoy, 1982); Peñas de Echauri/Navarra (Mateo \& Campoy, 1983); Bardenas/Navarra (Jordana et al., 1987).

REMARKs: Monreal and Campoy (1982) and Mateo and Campoy (1983) provided measurements and/or illustrations of the material collected in Navarra.

terrestris, Teratocephalus (Bütschli, 1873) De Man, 1876

SYNONYMY: Anguillula terrestris Bütschli, 1873; Teratocephalus angustus Massey, 1974.

Distribution: Sierra de Albarracín/Teruel (Gadea, 1952b); Sierra de las Nieves/Málaga (Gadea, 1953a); Desfiladero de Veyos and Picos de Europa/Asturias, and Las Salas/León (Gadea, 1953b); Sierra de Guadarrama/Madrid (Gadea, 1953c); Sierra Segundera/Zamora (Gadea, 1954a); Peña Trevinca/Zamora (Gadea, 1954b); Cueva Calera/Logroño (Gadea, 1955a); Moaña /Pontevedra (Gadea, 1955b); Selva de Oza and Panticosa/Huesca and Engors/Gerona (Gadea, 1956); Estany Llebreta/Lérida (Gadea, 1962b); Montejo de la Sierra/Madrid, and Sierra de Ronda/Málaga (Jiménez-Millán et al., 1965); Sierra de Cazorla/Jaén, Sierra de Cuenca/Cuenca and Sierra de Albarracín (Gadea, 1979); Sierras de Gata and Béjar/Salamanca (Palomo, 1979); Catalonian Central Pyrenean (Gadea, 1982); Quinto Real/Navarra (Monreal \& Campoy, 1982); Peñas de Echauri/Navarra (Mateo \& Campoy, 1983); Montes Alberes/Gerona (Gadea, 1983); Sansoain/Navarra (Hernández et al., 1988; Hernández \& Jordana, 1990 \& 1992); Sierra de
Grazalema/Cádiz (Santos-Lobatón, 1991); Sierra Morena/Sevilla (Santos-Lobatón, 1992); Añorbe, Beorburu, Ezprogui and Leoz (Armendáriz et al., 1996).

REMARKS: This is a cosmopolitan species. Some doubt exists on the identity of the specimens identified by Gadea (1952b, 1953a) due to its anterior vulva compared with the usual range of the species $(\mathrm{V}=44-45$ vs $\mathrm{V}=52-56)$. Anyway, posterior records indicate $T$. terrestris is a member of the Iberian fauna. Monreal and Campoy (1982) and Mateo and Campoy (1983) provided measurements and/or illustrations of the material collected in Navarra.

\section{terricola, Rhabditis Dujardin, 1845}

SynONYMY: Angiostoma terricola (Dujardin, 1845) Diesing, 1851; Leptodera terricola (Dujardin, 1845) Chatin, 1888; Rhabditis terricola spiculofusa Abuladze, 1934; Rhabditis aspera Bütschli, 1873; Rhabditis (Choriorhabditis) aspera Bütschli, 1873 (Osche, 1952)

Distribution: Sierra Nevada/Granada (MuñozMedina, 1945); Onda/Castellón (Gadea, 1952c); Peña Prieta/León-Cantabria-Palencia (Gadea, 1953b); Selva de Oza, Panticosa, Circo de Soaso and Turbón/Huesca (Gadea, 1956); Fornells and San Luis/Menorca-Balerares (Gadea, 1962a); Central Spain (Jiménez-Millán, 1962); Isla Mayor/Murcia (Gadea, 1976); Sierras de Gata and Béjar/Salamanca (Palomo, 1979); Catalonian Central Pyrenean (Gadea, 1982); Quinto Real/Navarra (Monreal \& Campoy, 1982); Quinto Real/Navarra (Mateo \& Campoy, 1983); Montes Alberes/Gerona (Gadea, 1983).

REMARKS: Spanish records of this species correspond to $R$. aspera, a junior synonym of $R$. terricola. Muñoz-Medina (1945; mentioned by Jiménez-Guirado, 1976a) recorded Rhabditophanes terricola, certainly (?) a typographical error refering $R$. terricola. Gadea (1952a) described and illustrated a male specimen. Gadea (1953b) recorded $R$. terricola but the author also refered to $R$. teres - which Gadea mentioned as a junior synonym of $R$. terricola - and this findind surely corresponds to the latter species. Monreal and Campoy (1982) and Mateo and Campoy (1983) provided measurements and/or illustrations of the material collected in Navarra.

tigrodon, Panagrolaimus Fuchs, 1930

SynONyMY: Pangrodontus tigrodon (Fuchs, 1930) Thorne, 1937; Panagrolaimoides tigrodon (Fuchs, 1930) Ivanova, 1958; Panagrodontus dentatus Thorne, 1935; Panagrolaimus dentatus (Thorne, 1935) Rühm, 1956; Panagrodontus ruehmi Ivanova, 1958. 
DistRIBUTION: Quinto Real/Navarra (Hernández \& Jordana, 1992).

REMARKS: No additional information about this species is available.

tripartitum, Cruznema (von Linstow, 1906) Zullini, 1982

SyNONYMY: Rhabditis tripartita von Linstow, 1906; Rhabditis (Cruznema) tripartita von Linstow, 1906 (Sudhaus, 1974); Rhabditis lambdiensis Maupas, 1919; Pelodera (Cruznema) lambdiensis (Maupas, 1919) Dougherty, 1953; Cruznema lambdiensis (Maupas, 1919) Thorne, 1961; Rhabditis monhysteroides Skwarra, 1921; Pelodera (Cruznema) monhysteroides (Skwarra, 1921) Dougherty, 1955; Rhabditis cryptocercoides Wollenweber, 1921; Rhabditis (Mesorhabditis) cryptocercoides (Wollenweber, 1921) Osche, 1952); Mesorhabditis cryptocercoides (Wollenweber, 1921) Dougherty, 1955; Rhabditis (Mesorhabditis) macrocheila Kreis, 1932; Mesorhabditis macrocheila (Kreis, 1932) Dougherty, 1955; Cruznema cruznema Artigas, 1927; Epimenides extricatus Gutiérrez, 1949; Pelodera melisi Marinari, 1957; Pelodera (Cruznema) melisi Marinari, 1957 (Meyl, 1961).

Distribution: Sierra Nevada/Granada (Picazo et al., 1988; Ocaña \& Picazo, 1991).

REMARKS: No additional information about this species is available.

tristis, Protorhabditis (Hirschmann,

Dougherty, 1955

SyNONYMY: Rhabditis (Protorhabditis) tristis Hirschmann, 1952.

Distribution: Sierras de Gata and Béjar/Salamanca (Palomo, 1979); Beunza, and Unzué/Navarra (Hernández et al., 1988; Hernández \& Jordana, 1988 \& 1992); Sansoain/Navarra (Hernández et al., 1988; Hernández \& Jordana, 1990 \& 1992; Jordana et al., 1987); Añorbe, Beorburu, Ezprogui and Leoz/Navarra (Armendáriz et al., 1996).

REMARKS: No additional information about this species is available.

troglophilus, Cephalobus Andrássy, 1967

DistRIBUTION: Sansoain/Navarra (Hernández et al., 1988; Hernández \& Jordana, 1992); Unzué /Navarra (Hernández et al., 1988; Hernández \& Jordana, 1990 \& 1992); Bardenas/Navarra (Jordana et al., 1987).

REMARKs: No additional information about this species is available.

tuerkorum, Bunonema Sachs, 1949

Distribution: Beunza/Navarra (Hernández et al., 1988; Hernández \& Jordana, 1990 \& 1992;
Armendáriz \& Hernández, 1992); Sansoain /Navarra (Jordana et al., 1987; Hernández et al., 1988; Hernández \& Jordana, 1990 \& 1992; Armendáriz \& Hernández, 1992); Añorbe and Beorburu /Navarra (Armendáriz et al., 1996).

REMARKS: Armendáriz and Hernández (1992) provided measurements and illustrations of the specimens collected in northern Spain.

vexilliger, Cervidellus (De Man, 1880) Thorne, 1937 Synonymy: Cephalobus vexilliger De Man, 1880; Cephalobus (Acrobeles) vexilliger De Man, 1880 (De Man, 1921); Acrobeles vexilliger (De Man, 1880) Thorne, 1925; Ypsylonellus vexilliger (De Man, 1880) Andrássy, 1984; Acrobeles serratus Thorne, 1925; Cervidellus serratus (Thorne, 1925) Thorne, 1937.

Distribution: Peñas de Echauri/Navarra (Mateo \& Campoy, 1983); Bardenas/Navarra (Jordana et al., 1987); Igüer and Sayerri Valleys/Huesca (De Ley et al., 1994); Sansoain and Unzué/Navarra (Hernández et al., 1988; Hernández \& Jordana, 1990 \& 1992); Añorbe/Navarra (De Ley et al., 1994; Armendáriz et al., 1996); Beorburu, Ezprogui and Leoz/Navarra (Armendáriz et al., 1996).

REMARKS: De Ley et al. (1994) recorded and described in detail this species that they identified as Cervidellus serratus, one of its junior synonyms.

\section{Discussion}

According to the expossed information the catalogue of rhabditid species forming part of the Spanish fauna (except those inhabiting the Canary Islands) includes eighty species belonging to forty genera and twelve families, that is, little more than one-fourth of the total world rhabditid genera are presented in the Iberian soils and freshwaters. However, these data should be considered as preliminary results and taken with caution due that various error sources exist. As mentioned in the introduction, no monographic study on rhabditid diversity in the Iberian Peninsula has been carried out so far, thus an increase of the inventory should be obtained in the future when these studies are developed. The information refering to forty-five species is merely chorological and/or faunistic, lacking detailed descriptions and revisions of this material. Moreover, some of the records date from the fifties and sixties of the past century and were not ever confirmed. Therefore, new surveys and collections will be necessary to ascertain the real 
presence of a high number of species in the Iberian geography. If these actions were undertaken, the number of taxa would be likely reduced.

\section{References}

ANDRÁSSY, I. 1983. A taxonomic review of the suborder Rhabditina (Nematoda: Secernentia). Éditions de l'ORSTOM. Paris. 241 pp.

ANDRÁssy, I. 1984. Klasse Nematoda. Gustav Fischer Verlag. Stuttgart. 509 pp.

ARIAS, M. \& JiMÉNEZ-MiLlÁN, F. 1968. Chitinotylenchoides mediterranensis n. gen., n. sp. $8^{\text {th }}$ International Nematology Symposium, Antibes, Seetember 1965: 34.

Arias, M. \& JimÉnEZ-Millán, F. 1973. Tylopharynx foetidus (Bütschli, 1874) Sachs, 1950 en el Levante español. Cuadernos de Ciencias Biológicas, 2: 5355.

Arias, M., Jiménez-Millán, F. \& LóPez-Pedregal, J.M. 1963. Resultados previos del estudio fitonematológico de cultivos agrícolas de Sevilla. Boletín de la Real Sociedad Española de Historia Natural, 61: 45-49.

Armendáriz, I. \& Hernández, M.A. 1992. Cuatro especies de la familia Bunonematidae Micoletzky, 1922 (Nematoda, Rhabdtida) encontradas en pinares de Pinus nigra en Navarra (norte de España). Miscel-lània Zoològica, 16: 7-12.

Armendáriz, I., HernándeZ, M.A. \& JordAnA, R. 1996. Temporal evolution of soil nematode communities in Pinus nigra forests of Navarra, Spain. Fundamental and applied Nematology, 19: 561-577.

Bello, A., Laborda, E. \& Alvira, P. 1973. Estudios realizados en España sobre los nematodos de los agrios. Boletín de la Real Sociedad Española de Historia Natural (Sección Biológica), 71: 17-59.

De Ley, P. \& Blaxter, M.L. (in press). Systematics position and phylogeny. In: D.E. LEE (Ed.), The Biology of Nematodes: 1-30. Harwood Academic Press, Reading, UK.

De Ley, P., De Ley, I.T. \& Mundo-Ocampo, M. 1998. Nematode Workshop Manual: Identification of freeliving Secernentea. Department of Nematology, University of California, Riverside (unpublished).

De Ley, P., Hernández, M.A. \& Agudo, J. 1994. A redescription of Ypsylonellus similis (Thorne, 1925) Andrássy, 1984, with descriptions of some related species (Nematoda: Cephalobidae). Fundamental and applied Nematology, 17: 1-16.

De Ley, P., SiddiQI, M.R. \& BoströM, S. 1993. A revision of the genus Pseudacrobeles Steiner, 1938 (Nematoda. Cephalobidae). Part 2. Subgenus Bunobus subgen. n., problematical species, discus- sion and key. Fundamental and applied Nematology, 16: 289-308.

GADEA, E. 1952a. Contribución al estudio de los nematodos libres terrestres y dulceacuícolas de la fauna española. Publicaciones del Instituto de Biología Aplicada, Serie Zoología, 5: 1-213.

GADEA, E. 1952b. Sobre algunos nematodos libres terrestres de la Sierra de Albarracín. Publicaciones del Instituto de Biología Aplicada, 11: 152-172.

GADEA, E. 1953a. Nematodos libres de la Serranía de Ronda. Publicaciones del Instituto de Biología Aplicada, 13: 129-153.

GADEA, E. 1953b. Nematodos libres terrestres de los Montes Cántabros. Publicaciones del Instituto de Biología Aplicada, 14: 71-108.

GADEA, E. 1953c. Nematodos libres terrestres de la Sierra de Guadarrama. Publicaciones del Instituto de Biología Aplicada, 15: 113-130.

GAdEA, E. 1954a. Sobre algunos nematodos muscícolas de la Sanabria. Publicaciones del Instituto de Biología Aplicada, 17: 51-63.

GADEA, E. 1954b. Nematodos dulceacuícolas de la Sanabria. Publicaciones del Instituto de Biología Aplicada, 18: 133-150.

GADEA, E. 1955a. Nota sobre algunos nematodos muscícolas de la Sierra de la Demanda. Publicaciones del Instituto de Biología Aplicada, 19: 5-12.

GADEA, E. 1955b. Nematodos dulceacuícolas de Galicia. Publicaciones del Instituto de Biología Aplicada, 20: 77-114.

GadeA, E. 1956. Nematodos de los Pirineos Centrales. Actes du II Congrès Inter. D’Etudes Pyrénéennes, 3: 124-146, Toulouse.

GADEA, E. 1962a. Nematodos brioedáficos de la isla de Menorca. Publicaciones del Instituto de Biología Aplicada, 33: 71-111.

GADEA, E. 1962b. Nematodos muscícolas y de agua dulce del "Parque Nacional de Aigües Tortes y San Mauricio" (Pirineos Centrales). Actas del III Congreso Internacional de Estudios Pirenaicos, 129138, Zaragoza.

GADEA, E. 1963. Sobre la nematofauna del río Carrión. Publicaciones del Instituto de Biología Aplicada, 35: 141-147.

GADEA, E. 1964a. Sobre la nematofauna muscícola de las islas Medas. Publicaciones del Instituto de Biología Aplicada, 36: 29-38.

GADEA, E. 1964b. Sobre la nematofauna muscícola y liquenícola de la islas Pitiusas. Publicaciones del Instituto de Biología Aplicada, 37: 73-93.

GADEA, E. 1969. La nématofaune muscicole et calcophile des îles Baléares et de Sardaigne. Rapports et Proces Verbaus des Reunions-Commision, 19: 799801. 
GadeA, E. 1974. Nematodos liquenícolas de Columbretes. Miscel-lània Zoològica, 3: 3-8.

GADEA, E. 1976. Nematodos liquenícolas de la isla Mayor (Mar Menor). Miscel-lània Zoològica, 3: 1318.

GADEA, E. 1979. Sobre la nematofauna muscícola de las Sierras de Cazorla y Ronda. Publicaciones del Departamento de Zoología, Barcelona, 4: 7-11.

GAdEA, E. 1982. Nematofauna muscícola de los Pirineos Centrales de Cataluña. Publicaciones del Departamento de Zoología, Barcelona, 7: 37-43.

GadeA, E. 1983. Nematofauna muscícola de los Pirineos Orientales. I. Montes Alberes. Publicaciones del Departamento de Zoología, Barcelona, 9: 45-51.

Hernández, M.A. 1990. Heterocephalobus pseudolatus n. sp. encontrada en Navarra, norte de España (Nematoda, Cephalobidae). Boletín de la Real Sociedad Española de Historia Natural, 85: 101106.

Hernández, M.A. \& Jordana, R. 1988. First descriptions of the male of two species of Teratocephalus De Man, 1876 (Nematoda: Teratocephalidae). Revue de Nématologie, 11: 423-428.

HERnÁndeZ, M.A. \& JoRdANA, R. 1990. Estudio cualitativo de la fauna de nematodos de los suelos de tres bosques de Quercus en Navarra, España. In: Homenaje al Profesor García de Viedma (E.T.S.I.M.): 63-85. Fundación Conde del Valle de Salazar. Madrid.

HERnÁNDEZ, M.A. \& JoRdANA, R. 1992. Estudio comparativo de la fauna de nematodos en cinco biotopos naturales. In: AlemANY, A. (Ed.). Historia Natural'91: 139-149. Universitat de les Illes Balears. 626 págs.

Hernández, M.A., Mateo, M.D. \& Jordana, R. 1988. Estudio comparativo entre grupos tróficos de los suelos de cinco bosques de Navarra (tres naturales y dos de repoblación). Actas II Congreso Mundial Vasco de Biología Ambiental. Tomo II: 323-335.

JiMÉNEZ-GuIRADO，D. 1974. Notas sobre la posición taxonómica del género Rhaditophanes Fuchs, 1930 (Nematoda: Rhabditida) con motivo del hallazgo de Rhabditophanes schneideri (Bütschli, 1873) T. Goodey, 1953 en Granada. Cuadernos de Ciencias Biológicas, 3: 103-109.

JiMÉNEZ-GuIRADO, D. 1976a. Nematodos del suelo en la Sierra Nevada Granadina y valles adyacentes. I. Antecedentes y catálogo de los géneros y especies citados anteriormente. Cuadernos de Ciencias Biológicas, 5: 35-45.

JiMÉNEZ-GuIRADO, D. 1976b. Nematodos del suelo en la Sierra Nevada Granadina y valles adyacentes. II. Resultados de un muestreo preliminar. Cuadernos de Ciencias Biológicas, 5: 47-52.
JiMÉNEZ-MILLÁN, F. 1962a. Influencia del pH en cultivos de Rhabditis (Nematoda). Boletín de la Real Sociedad Española de Historia Natural, 60: 77-80.

JimÉnEZ-Millán, F. 1962b. Estudio de las especies transmisoras de virus vegetales Criconemoides xenoplax y Xiphinema index (Nematoda), nuevas en los viñedos españoles. Revista Ibérica de Parasitología, 22: $305-318$

Jiménez-Millán, F., Arias, M., Bello, A. \& LÓPEZPedregal, J.M. 1965. Catálogo de los nematodos fitoparásitos y peri-radiculares encontrados en España. Boletín de la Real Sociedad Española de Historia Natural, 63: 47-104

Jordana, R., Arbea, J.I., Moraza, L., Montenegro, E., MATEO, M.D., HernÁNDEZ, M.A. \& Herrera, L. 1987. Effect of refforestation by conifers in natural biotopes of middle and South Navarra (Norhtern Spain). Revue suisse de Zoologie, 94: 491-502.

Karegar, A., De Ley, P. \& Geraert, E. 1997. A detailed morphological study of Acromoldavicus skrjabini (Nesterov \& Lisetskaya, 1965) Nesterov, 1970 (Nematoda: Cephaloboidea) from Iran and Spain. Fundamental and applied Nematology, 20: 277283

MAdrid-Moreno, J. 1911. Datos para el estudio del plancton del río Lozoya. Boletín de la Real Sociedad Española de Historia Natural, 11: 173-176.

MAteO, M.D. \& CAMPoy, A. 1983. Estudio de los Nematodos libres de las Peñas de Echauri (Navarra). Publicaciones de Biología de la Universidad de Navarra, Serie Zoológica, 9: 1-64.

Monreal, J.I. \& CAMPoY, A. 1982. Estudio faunístico del macizo de Quinto Real. VI: Nematodos (Nematoda). Publicaciones de Biología de la Universidad de Navarra, Serie Zoológica, 8: 1-92.

MuÑOZ-MedinA, J.M. 1945. Algunos helmintos libres y fitoparásitos de la región granadina. Revista Ibérica de Parasitología, Tomo extraordinario: 246-250.

NAVACERRADA, G. 1975. Nematodos asociados a los viñedos españoles. Boletín de la Real Sociedad Española de Historia Natural, 73: 47-56.

NaVarro, P. \& Lluch, J. 1999. Paracrobeles psammophilus sp. nov. (Nematoda: Cephalobidae) from El Saler, Valencia (Spain). Journal of Zoology, London, 249: 481-485.

OCAÑA, A. 1992. Relationship between nematode species and physico-chemical characteristics of spring water. III: pH, oxygen and iron. Nematologia mediterranea, 20: 181-186.

OCAÑA, A. \& PicAzo, J.S. 1991. Study on nematode species encountered in the Monachil River (Granada, Spain): Response to organic pollution. Verhandlungen - Internationale Vereinigung für Theoretische und Angewandte Limnologie, 24: 2729-2737. 
Picazo, J., OcAÑA, A. \& JiméneZ-Millán, F. 1989. Grupos tróficos de nematodos en el bentos del río Monachil (Granada). Miscelánea zoológica, 13: 111.

SANTOS-LoBATÓN, M.C. 1991. Sobre la nematofauna muscícola del pinsapar (Abies pinsapo Boiss.) de la Sierra de Grazalema (España). Nematologia mediterranea, 19: 197-206.

SANTOS-LoBATÓN, M.C. 1992. Sobre la biocenótica de los nematodos muscícolas. In: Alemany, A. (Ed.). Historia Natural'91: 151-161. Universitat de les Illes Balears. 626 págs.
Shahina, F. \& De Ley, P. 1997. Two new species of Cephalobidae from Valle de la Luna, Argentina, and observations on the genera Acrobeles and Nothacrobeles (Nematoda: Rhabditida). Fundamental and applied Nematology, 20: 329-347.

Recibido, el 10-IV-2001

Aceptado, el 15-VI-2001 Publicado, el 00-XX-2001 
Appendix I.- Taxonomic position of rhabditid species found in peninsular Spain and Balearic Islands.

\section{ORDEN RHABDITIDA Chitwood, 1933}

\section{SUBORDEN CEPHALOBINA Andrássy, 1974}

Superfamilia Cephaloboidea Filipjev, 1934

Familia Cephalobidae Filipjev, 1934

Subfamilia Acrolobinae De Ley, Boström et Siddiqi, 1993 Acrolobus Boström, 1985

$$
\text { A. emarginatus }
$$

Subfamilia Cephalobinae Filipjev, 1934

Acrobeles von Linstow, 1877

A. ciliatus

A. complexus

A. elaboratus

A. singulus

Acrobeloides Cobb, 1924

A. buetschlii

A. nanus

Cephalobus Bastian, 1865

C. labiatus

C. parvus

C. persegnis

C. troglophilus

Cervidellus Thorne, 1937

C. cervus

C. neftasiensis

C. vexilliger

Chiloplacus Thorne, 1937

$$
\begin{aligned}
& \text { C. minimus } \\
& \text { C. propinquus } \\
& \text { C. symetricus }
\end{aligned}
$$

Eucephalobus Steiner, 1936

E. cornis

E. mucronatus

E. oxyuroides

E. paracornutus

E. striatus

Heterocephalobus (Brzeski, 1960) Brzeski, 1961

H. bisimilis

H. elongatus

H. latus

Paracrobeles Heyns, 1968

P. psammophilus

Pseudacrobeles Steiner, 1938

P. (Pseudacrobeles) Steiner, 1938

P. (P.) teres

P. (Bunobus) De Ley, Boström et Siddiqi, 1993

P. (B.) loofi

P. (B.) pseudolatus

Stegelletina Andrássy, 1984

S. devimucronata

S. similis

Familia Elaphonematidae Heyns, 1962

Subfamilia Kirjanoviinae Andrássy, 1976

Acromoldavicus Nesterov, 1970

$$
\text { A. skrjabini }
$$

Familia Osstellidae Heyns, 1962

Subfamilia Drilocephalobinae Ali, Suryawanshi et Chisty, 1973

Drilocephalobus Coomans et Goodey, 1965

$$
\text { D. coomansi }
$$

Superfamilia Panagrolaimoidea Thorne, 1937

Familia Panagrolaimidae Thorne, 1937

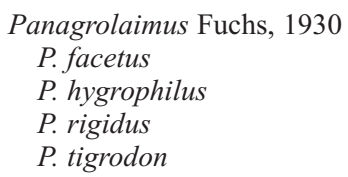

Superfamilia Strongyloidoidea Chitwood et McIntosh, 1934 Familia Alloionematidae Chitwood et McIntosh, 1934

Rhabditophanes Fuchs, 1930

R. schneideri

SUBORDEN RHABDITINA Andrássy, 1974

Superfamilia Bunonematoidea Micoletzky, 1922

Familia Bunonematida Micoletzky, 1922

Subfamilia Bunonematinae

Bunonema Jägerskiold, 1905

B. multipapillatum

B. penardi

B. reticulatum

B. richtersi

B. tuerkorum

Rhodolaimus Fuchs, 1930

R. goffarti

Superfamilia Rhabditoidea Örley, 1880

Familia Rhabdiditidae Örley, 1880

Cruznema Artigas, 1927

$$
\text { C. tripartitum }
$$

Cephaloboides Rahm, 1928

C. curvicaudata

Poikilolaimus Fuchs, 1930

P. oxycerca

Diploscapter Cobb, 1913

D. coronatus

Mesorhabditis Osche, 1952

M. spiculigera

Pellioditis Dougherty, 1953

P. pellio

P. pellioides

Pelodera Schneider, 1866

P. punctata

P. strongyloides

P. teres

Phasmarhabditis Andrássy, 1976

P. papillosa

Protorhabditis Osche, 1952

P. filiformis

P. tristis

Rhabditella Cobb, 1929

R. pseudoelongata

Rhabditis Dujardin, 1845

R. axei

R. longicaudata

R. maupasi

R. producta

R. terricola

Rhabditoides Goodey, 1929

R. inermis

SUBORDEN DIPLOGASTERINA Micoletzky, 1922

Superfamilia Diplogasteroidea Micoletzky, 1922

Familia Diplogasteroididae Filipjev et Schuurmans Stekhoven,

1941 
Rhabditidoides Rahm, 1928

R. stigmatus

Familia Diplogasteridae Micoletzky, 1922

Diplogaster Schultze in Carus, 1857

D. rivalis

Diplogasteritus Paramonov, 1952

D. nudicapitatus

Metadiplogaster Meyl, 1961

M. graciloides

Familia Neodiplogasteridae Paramonov, 1952

Fictor Paramonov, 1952

F. fictor

Mononchoides Rahm, 1928

M. ruffoi

Oigolaimella Paramonov, 1952

O. carinata

Pristionchus Kreis, 1932

P. lheritieri

Familia Tylopharyngidae Filipjev, 1934

Tylopharynx De Man, 1876

T. foetida
SUBORDEN TERATOCEPHALINA Andrássy, 1974

Superfamilia Teratocephaloidea Andrássy, 1958

Familia Teratocephalidae Andrássy, 1958

Subfamilia Teratocephalinae Andrássy, 1958

Teratocephalus De Man, 1876

T. costatus

T. dadayi

T. palustris

T. subvexus

T. terrestris

Subfamilia Metateratocephalinae Eroshenko, 1973

Metateratocephalus Eroshenko, 1973

M. crassidens

Appendix II. - Species list of rhabditid nematodes found in peninsular Spain and Balearic Islands

\author{
Acrobeles ciliatus \\ Acrobeles complexus \\ Acrobeles elaboratus \\ Acrobeles singulus \\ Acrobeloides buetschlii \\ Acrobeloides nanus \\ Acrolobus emarginatus \\ Acromoldavicus skrjabini \\ Bunonema multipapillatum \\ Bunonema penardi \\ Bunonema reticulatum \\ Bunonema richtersi \\ Bunonema tuerkorum \\ Bursilla paucipapillata \\ Cephalobus labiatus \\ Cephalobus parvus \\ Cephalobus persegnis \\ Cephalobus troglophilus \\ Cervidellus cervus \\ Cervidellus neftasiensis \\ Cervidellus vexilliger \\ Chiloplacus minimus \\ Chiloplacus propinquus \\ Chiloplacus symetricus \\ Cruznema tripartitum \\ Cephaloboides curvicaudata \\ Diplogaster rivalis \\ Diplogasteritus nudicapitatus \\ Diploscapter coronatus \\ Drilocephalobus coomansi
}

\author{
Eucephalobus cornis \\ Eucephalobus mucronatus \\ Eucephalobus oxyuroides \\ Eucephalobus paracornutus \\ Eucephalobus striatus \\ Fictor fictor \\ Heterocephalobus bisimilis \\ Heterocephalobus elongatus \\ Heterocephalobus latus \\ Mesorhabditis spiculigera \\ Metadiplogasteroides graciloides \\ Metateratocephalus crassidens \\ Mononchoides ruffoi \\ Oigolaimella carinata \\ Panagrolaimus facetus \\ Panagrolaimus hygrophilus \\ Panagrolaimus rigidus \\ Panagrolaimus tigrodon \\ Paracrobeles psammophilus \\ Paroigolaimella bernensis \\ Pellioditis pellio \\ Pellioditis pellioides \\ Pelodera punctata \\ Pelodera strongyloides \\ Pelodera teres \\ Phasmarhabditis papillosa \\ Poikilolaimus oxycerca \\ Pristionchus lheritieri \\ Protorhabditis filiformis \\ Protorhabditis tristis
}

Pseudacrobeles (Bunobus) loofi

Pseudacrobeles (Bunobus) pseudolatus

Pseudacrobeles (Pseudacrobeles) teres

Rhabditella pseudoelongata

Rhabditidoides stigmatus

Rhabditis axei

Rhabditis longicaudata

Rhabditis maupasi

Rhabditis producta

Rhabditis terricola

Rhabditoides inermis

Rhabditophanes schneideri

Stegelletina devimucronata

Stegelletina similis

Teratocephalus costatus

Teratocephalus dadayi

Teratocephalus palustris

Teratocephalus subvesus

Teratocephalus terrestris

Tylopharynx foetida 